\title{
La estrategia de educación permanente en salud como posibilidad de intervención social
}

\author{
Marina Arruda P.* \\ Geraldo Locks A.** \\ Fernando Pagliosa L.*** \\ Aliete Araújo P.
}

RESUMEN

Este artículo presenta una experiencia acerca de intervención social que tuvo como objetivo capacitar a los profesionales de la salud para trabajar en las políticas del Sistema Único de Salud (SUS). La metodología de la Educación Permanente en Salud (EPS) fue la estrategia utilizada para el trabajo que resultó en la planificación de acciones y en la mejoría de la calidad de los servicios prestados a la población. Buscando dar nuevas directrices y delinear nuevas posibilidades de intervención social, este artículo muestra una experiencia que se asocia a este esfuerzo de transformación de las prácticas.

Palabras clave: Integración enseñanza - servicio de salud - estrategia de educación permanente - intervención social.

\section{A estratégia de educação permanente em saúde como possibilidade de intervenção social}

\section{RESUMO}

Este artigo apresenta um relato de experiencia sobre a intervenção social que teve como objetivo capacitar os profissionais da saúde para um trabalho pautado nas políticas do Sistema Único de Saúde (SUS). A metodologia da Educação Permanente em Saúde (EPS) foi a estratégia utilizada para

* Arruda, Marina Patrício. Socióloga. Magíster y Doctora en Servicio Social. Profesora y Coordinadora del Programa de post graduación en Salud Colectiva-Máster-UNIPLAC) Lages-SC, miembro del Grupo de Educación Permanente (EDUPE) del Curso de medicina. Correo electrónico: marininh@uniplac.net ou marininh@terra.com.br

** LOCKS, Geraldo Augusto, Sociólogo. Magíster y doctor en Antropología Social. Profesor y miembro del Grupo de Educación Permanente (EDUPE) del Curso de Medicina/ UNIPLAC. Correo electrónico: geraldo@uniplac.net

*** PAGLIOSA, Fernando Luiz. Médico. Magíster en salud colectiva. Profesor y miembro del Grupo de Educación Permanente (EDUPE) del Curso de Medicina/UNIPLAC. Correo electrónico: pagliosa@uniplac.net

**** ARAÚJO, Aliete Perin. Pedagogo. Magíster en Sociología Política. Profesora y miembro del Grupo de Educación Permanente (EDUPE) del curso de Medicina/UNIPLAC. Correo electrónico: alietepe@terra.com.br 
o trabalho que resultou no planejamento de ações e na melhoria da qualidade dos serviços prestados à população.

Palavras-chave: integração ensino-serviço de saúde - Estratégia da Educação Permanente - intervenção social

\section{The lifelong learning strategy in health and social intervention can}

\section{ABSTRACT}

This article presents an experience of social intervention, in which the objective was to train health professionals to work on the policies of the Unified Health System (SUS). The methodology of Continuing Education in Health (EPS) was the strategy used for work, which resulted in action planning and improving the quality of services rendered to the population. This article seeks to provide new guidelines and outline new opportunities for social intervention. The paper shows an experience that is associated with this effort to transform the practices.

Key words: Integration education - health services - lifelong learning strategy - social intervention 


\section{Introducción}

La articulación servicio de salud - universidad, sigue la misma dirección del debate institucional que en la actualidad busca desarrollar en la práctica una forma más adecuada de colaboración y de contribución para la organización de los servicios de salud de los municipios. La práctica es mirada, generalmente, como un campo únicamente de aplicación o verificación de ideas o modelos. Tal mirada, niega la responsabilidad social de la universidad y de sus investigadores en lastransformaciones sociales, políticas económicas y educacionales para los temas de Salud. La integración enseñanzaservicio de salud se presenta entonces, como fundamental para la transformación social, evidenciando la posibilidad de intervención de la Universidad en la sociedad donde se inserta, no sólo generando conocimiento, sino que colocando este conocimiento también al servicio de la comunidad.

La búsqueda de nuevos referentes para la intervención del Trabajo Social, apunta hacia nuevas posibilidades de transformación de la realidad social, debiendo distanciarse de concepciones simplificadas todavía hegemónicas en el entendimiento del proceso salud -enfermedad. Para que ocurra este cambio, el protagonismo de los profesionales de la salud es fundamental en la determinación de dinámicas en el campo de la salud. Una práctica profesional centrada en la especialización y en un abordaje reduccionista, no logra enfrentar las necesidades sociales, como tampoco comprende la naturaleza de los problemas de salud que cotidianamente vive la población.

En tal sentido, en Brasil en las dos últimas décadas, se han producido algunos movimientos de cambio, tanto en el sector de la salud (la implementación del Sistema Único de Salud - SUS), como en el sector de la educación, que corresponde a la formación para el área de la salud.

En este contexto se desarrolló la intervención social llamada "Organización de servicios de salud: un desafío de educación permanente", que fue ejecutada a lo largo del año 2006 y 2007 por el Grupo de Educación Permanente (EDUPE) del Curso de Medicina de la Universidad de Planalto Catarinense (UNIPLAC), por medio de acciones pedagógicas que buscaban una reflexión crítica acerca de los temas cotidianos del trabajo en salud. Este proyecto de 
extensión posibilitó una serie de aprendizajes para la reformulación de prácticas, teniendo presente la cercanía con los problemas de una realidad social específica.

La metodología centrada en la estrategia de la Educación Permanente en Salud (EPS) orientó la organización de los servicios municipales de salud del pequeño municipio de la región Medio-Oeste del Estado de Santa Catarina. Partimos de la base que la organización de los servicios de salud, implica la adopción de una perspectiva cualitativa de renovación y transformación de las prácticas profesionales. Esta orientación pedagógica se constituye en un instrumento de desarrollo de la ciudadanía, capaz de favorecer la construcción de un ambiente efectivo de cambio. Este proyecto tuvo por objetivo capacitar a los profesionales de la salud de São Cristóvão do Sul SC, para un trabajo pautado en las políticas del Sistema Único de Salud (SUS) contribuyendo de este modo al mejoramiento de la calidad de los servicios prestados a la población.

\section{Revisando la literatura acerca de la educación permanente y educación continua}

La Educación Permanente en Salud (EPS) es una estrategia fundamental para la transformación del trabajo en el sector de la salud (Cecim, 2004). Esta estrategia pedagógica puede ser difundida "por dentro de los sectores" volviéndose orgánica y fundadora de reflexiones críticas y de discusiones técnicas capaces de incrementar la práctica de los profesionales de la salud. Esta acción nos permite articular las orientaciones del Sistema Único de Salud al cotidiano profesional.

La Educación Permanente en Salud se presenta como una propuesta de acción estratégica capaz de contribuir a la transformación de los procesos formativos, de las prácticas pedagógicas y de la salud (Brasil, 2004). La idea es que los profesionales de la salud se aproximen cada vez más a los pacientes, tomando en cuenta las necesidades del lugar en que ellos viven. Este proceso parte del supuesto de que para aprender, no basta la transferencia de conocimiento, sino que es necesario crear un ambiente propicio a la construcción orgánica y compartida del conocimiento. En esta orientación, el "escuchar" se vuelve más importante que el "hablar". El buen escuchador habla y dice su posición con desenvoltura (Freire, 2000). 
Para una mejor comprensión del direccionamiento de esta intervención social, conviene distinguir, para inmediatamente articular, la educación Permanente (EP) y Educación Continua (EC). La idea es que se comprenda la EC como un recurso utilizado para dar cuenta de necesidades más específicas y teóricas, utilizando de forma más puntual y estructurada los contenidos. Ello porque, muchas veces, a lo largo del proceso de EP, surge la necesidad de comprensión de nuevos contenidos y en este sentido, los momentos de EC son esenciales para llenar vacíos e incomprensiones conceptuales.

Aprender a aprender dentro de la perspectiva de la educación permanente, es un tipo de aprendizaje orgánico, que soporta correcciones, modificaciones y adaptaciones dinámicas, siendo esta la base del proceso de auto-renovación capaz de producir cambios profundos en los seres vivos. En la reforma del pensamiento, la estrategia va a generar un pensamiento que une y enfrenta la incerteza (Morin, 2000).

Educación permanente y educación continua son momentos diferentes de un mismo proceso de aprendizaje, juntos sintetizan lo que Morin (2000) llama dialógica; procesos que son al mismo tiempo antagónicos, concurrentes y complementarios. A partir de esta comprensión, el Grupo de Educación Permanente (EDUPE) vio la necesidad de construcción de un espacio sistemático para cada uno de estos momentos. El espacio de la educación permanente pasó a ser considerado como estrategia de renovación de la práctica, por medio de la reflexión y de la relación de intercambio entre los profesionales de la salud. Mientras que la educación continua se consideró como programa, es decir, como un espacio para retomar contenidos, conceptos importantes para la retroalimentación de la práctica profesional.

El análisis de programas establecidos a priori articulados a las estrategias de acción, permitió avanzar en la reforma de nuestro pensamiento y comprender que cualquier separación entre educación permanente y educación continua, implica una fragmentación del proceso educativo, ya que una es prolongación de la otra. "La Educación Permanente en Salud todavía configura para otros educadores, el desdoblamiento, sin conexión, de varios movimientos de cambio en la formación de los profesionales de la 
salud, resultado del análisis de las construcciones pedagógicas de la educación en servicios de salud" (Cecim, 2004: 162).

Cabe destacar que la humanización del SUS puede ser tomada de forma general como un cambio en la mirada de los profesionales, en el momento de prestar servicios a los usuarios (Brasil, 2004). Esta nueva perspectiva, representa un aumento del grado de compromiso con respecto a la atención y al servicio como un todo, indicando la co-responsabilidad de cada uno. En este sentido, la EPS como espacio esencialmente reflexivo, posibilita una mirada compartida de responsabilización de los involucrados.

Finalmente, las reformulaciones del SUS imponen cambios que implican un compromiso con la colectividad. Cambian las relaciones interpesonales y el proceso de trabajo. El diálogo y la escucha, la construcción de vínculo y la acogida, han sido dispositivos relevantes en las propuestas de humanización de la salud como muestran diversos autores (Silva Júnior et al., 2003; Teixeira, 2003). Crece, por lo tanto, la importancia de experiencias localizadas de experimentación de la EPS como un instrumento de cambio y transformación social.

\section{Pasos metodológicos utilizados en la intervención}

Este proyecto de intervención se caracterizó por una composición metodológica de naturaleza cualitativa, en la que se seleccionó como principal actor al equipo de salud del municipio de São Cristóvão do Sul SC, con sus dinámicas y necesidades.

El primer paso de este proceso fue la distribución de los profesionales de la salud, en cuatro grupos interdisciplinarios de aproximadamente 10 personas, que se reunían quincenalmente con la presencia de un mediador del EDUPE, acompañado de una estudiante del Curso de Servicio Social de la UNIPLAC. Correspondió al mediador dirigir los debates, elaborar las síntesis de las discusiones, e incentivar a los integrantes para describir sus prácticas profesionales. A lo largo de estas reuniones de EPS surgió la necesidad de profundizar a través de estudios adicionales, individuales o en grupo, utilizando para ello talleres, seminarios, mesa de discusiones y/u otras actividades que permitieran el discernimiento de los problemas apuntados. 
Cada mediador, con la colaboración del estudiante becado', medió y elaboró informes semanales acerca de los problemas, buscando siempre soluciones y abordajes teóricos que asegurasen una reflexión consistente.

La propuesta se inició con la comprensión del proceso de Educación Permanente en Salud: sus conceptos, supuestos, objetivos. En este camino, las discusiones fueron importantes para la comprensión del concepto de EPS como un proceso de reflexión sobre la práctica y consecuente distinción del concepto de EC. Esta aclaración fue oportuna para la elaboración de estrategias colectivas y adecuadas para la planificación de los trabajos.

La importancia de la colaboración entre el municipio y la universidad se volvió fundamental para la cualificación de los profesionales involucrados, considerando que la atención a las necesidades de salud de la población, no se restringe al tratamiento de enfermedades o recuperación de las mismas, sino que se extiende a la promoción de la salud y principalmente, a la comprensión de un nuevo paradigma en salud. Igualmente importante fue el diagnóstico inicial, realizado por el EDUPE, referente a las condiciones del municipio para el desarrollo del proyecto, considerando principalmente su estructura administrativa, instalaciones físicas, situación financiera y el número de habitantes. Se destaca la decisión política de la gestión pública municipal como pilar de la propuesta de cambio en el área de la salud del referido municipio.

Las actividades se centraron en torno a la realidad del trabajo cotidiano y en la problematización de las vivencias de los profesionales involucrados. Ellos, en permanente interacción con informaciones, teorías, discusiones y talleres, buscaban la transformación del proceso de trabajo cotidiano. De acuerdo con lo planificado, los encuentros para el desarrollo de este proceso se realizaron quincenalmente. En las semanas en que los mediadores de EDUPE se ausentaron, los profesionales recibían tareas para ser cumplidas. Tales tareas se relacionaban con las necesidades que emergían de las discusiones precedentes, generalmente acompañadas de textos para lecturas, buscando el asentamiento teórico para las discusiones subsecuentes. Se destaca el modo

1 Estas becas integran el Programa de Asistencia Social de la UNIPLAC. 
como los profesionales cumplieron estas tareas, la dedicación en la construcción de la autonomía y gerencia responsable de su propio proceso de trabajo.

\section{Resultados y discusión}

El fortalecimiento de las relaciones interpersonales propició la construcción de vínculos y favoreció un trabajo en grupo, sustentado en principios y valores éticos. Pues, no existe conocimiento profesional riguroso basado en la racionalidad técnica, que dé cuenta de la actuación en el ámbito imprevisible y complejo de las relaciones interpersonales de las profesiones de la salud. Especialmente si tal conocimiento no se articula a la práctica y a la realidad, teniendo en cuenta actitudes y competencias favorables al restablecimiento de la confianza mutua y empatía en las relaciones educador-educando y profesional de la salud- paciente (Komatsu, 1999).

A modo general, la mayor parte de los participantes de esta intervención social, de forma individual o colectiva, discutió contenidos, los relacionó con la realidad del municipio y propuso modificaciones para sus prácticas cuando lo juzgó importante. Algunos tuvieron dificultades relativas a la lectura e interpretación de los textos, lo que comprometió algunas veces, su participación en los debates. En ciertos momentos, los participantes se preocuparon solamente de responder las cuestiones presentadas, sin levantar cuestionamientos relacionados a su trabajo, lo que implicó un tiempo mayor de elaboración para la transformación de su práctica.

Los grupos demostraron una gran capacidad de auto-organización, eligiendo democráticamente a los coordinadores de las reuniones, elaborando los relatos, explorando adecuadamente los textos indicados para la discusión de las cuestiones propuestas. En la realización de las tareas, percibieron las necesidades de conocimientos adicionales para la mejoría de su trabajo cotidiano, teniendo en cuenta la organización de los servicios y la planificación de las acciones de salud. Concluyeron que la fragmentación de la formación de los profesionales de la salud y la dificultad de trabajar de forma interdisciplinaria, obstaculizan la reflexión conjunta acerca de la práctica y necesidad de cambio. 
El esfuerzo de algunas personas para superar dificultades específicas, sin embargo, fundamentales para el proceso en curso, sorprendió a todos. Frente a las dificultades de lectura e interpretación de textos, ellos mismos buscaron ayuda en sus familias y colegas para la superación de estos límites. Tal actitud, despertó la sensibilidad y la solidaridad entre el grupo, fortaleciendo la construcción de vínculos y de respeto a las diferencias ahí percibidas.

Se destaca en especial, un momento en que los profesionales vivenciaron la declaración sincera y feliz de una funcionaria que había iniciado su proceso de alfabetización. La expresión de su gratitud por la buena voluntad y disponibilidad de una colega de trabajo que se propuso en los días sábados por la tarde, enseñarle a leer y escribir, conmovió a todos. Otras tantas declaraciones nos hicieron dar cuenta que el proceso de EPS los motivó a retomar los estudios, a buscar informaciones y a ampliar sus conocimientos.

La gran contribución de la EPS para el proceso de trabajo, es con respecto a los cambios que fueron siendo observados en el ambiente laboral, es decir, hubo impactos inmediatos en la práctica profesional. La estrategia de acogida como manera de actuar, traspasó el proceso de renovación y transformación de las prácticas profesionales arraigadas en São Cristóvão do Sul, permitiendo que el EDUPE pusiera en marcha una interesante acción pedagógica.

Acoger a las personas, significa distanciarse de la atención exclusivamente tecnocrática, para permitir la atención humanizada y sensible. Ello sería el traspaso fundamental operado por la propia noción de acogida (Silveira et al., 2005). La importancia de una propuesta pedagógica consolidada en la estrategia de la acogida, se sustentó en el establecimiento del vínculo entre el grupo que promovía esta intervención comunitaria y los profesionales de la salud, influyendo de este modo en las prácticas observadas a lo largo del desarrollo de esta intervención.

Muchos profesionales destacaron que, a partir de las reflexiones en los grupos, buscaron cambiar su práctica, poniendo más atención en su colega de trabajo, percibiendo la necesidad del trabajo en equipo, de decisiones conjuntas, de ayudar al colega cuando éste estuviera sobrecargado, de buscar saber lo que los demás piensan sobre su trabajo y de conocer mejor el trabajo del compañero. Constataron que el trabajo realizado hasta entonces, no incluía 
colaboración de colegas, y que deberían aprender a actuar de forma colaborativa, dejando a un lado el orgullo y el miedo de "perder espacio", pues el trabajo final sería siempre colectivo. Percibieron que muchas veces faltaba unión en el grupo, y que criticaban el trabajo del otro sin saber lo que realmente hacía. Varias reflexiones permitieron que angustias y fragilidades fuesen discutidas por el grupo, principalmente referidas a la importancia de aprender a hacer y recibir críticas de forma constructiva, con respeto y ética.

El consenso de que la EPS adoptada por el EDUPE fue una forma diferente de orientar reflexiones y capacitar para el trabajo, emergió de forma natural. Entendieron rápidamente que el trabajo en salud debiera ser realizado de forma conjunta, con responsabilidad compartida que estimulase la manifestación de todos en la "escucha" de cada uno del equipo de trabajo. Esta estrategia funcionó como estímulo para que muchos se empeñaran en aprender, estudiar y buscar nuevos conocimientos. Algunos fueron testigos de que se quedaron con más coraje para hablar de sí mismos, y para exponer sus opiniones en el debate grupal. Se sintieron sujetos a su trabajo y percibieron la importancia de informar a los usuarios de la Unidad Básica de Salud, acerca de los encuentros de EPS en los que el consultorio permanecía cerrado por la mañana.

En ese sentido, destacan la creación de varias estrategias de comunicación con los pobladores, utilizadas para que pudieran explicar que este día estaba reservado para "un curso de capacitación que traería mejoras al servicio de salud del municipio". Nos contaron después, que los pobladores acataron con tranquilidad los argumentos presentados y que algunos usuarios llegaron a preguntar si podrían participar también de estas reuniones. Esta información muestra la comprensión de la comunidad, en relación a la necesidad de innovación de la práctica profesional y justifica el esfuerzo de integración enseñanza-servicio.

\section{Consideraciones finales}

Capacitar a los profesionales de la salud como sujetos sociales, responsables por la mediación de los derechos en salud, implicó la organización de espacios de reflexión sobre las prácticas desde la lógica de la Educación Permanente en Salud, es decir, en la búsqueda de soluciones a partir de los problemas enfrentados en 
el cotidiano del ámbito laboral, teniendo presentes las experiencias de cada uno.

Los profesionales con los que interactuamos, constataron que aun cuando trabajaran juntos, no se conocían, no sabían de sus historias, ni de sus problemas, ni de sus dudas con respecto al trabajo ejecutado. Evaluaron a los profesionales y concluyeron que éstos fallan en la atención a la comunidad y que necesitan conversar más para exponer sus experiencias y dudas, de manera que amplíen su propia mirada acerca de lo que ocurre en el lugar de trabajo.

Los principales avances de esta intervención fueron alcanzados por medio de una metodología de trabajo, cuyo mayor desafío fue desarrollar la capacidad de aprender a aprender. Este proceso hace referencia a los aprendizajes que el individuo realiza por sí mismo, en los que se ausenta la transmisión de conocimientos y se relaciona con la búsqueda de una educación fundamentada en la autonomía, en el respeto a la diferencia y en la construcción de vínculos.

Esta intervención se justificó al propiciar transformaciones fundamentales en la atención a los pobladores, el desarrollo de la capacidad de aprendizaje y de habilidades profesionales para el enfrentamiento creativo de situaciones cotidianas. La EPS, entendida como aprendizaje, donde el aprender se incorpora a lo cotidiano de las organizaciones y al trabajo, transformó las prácticas centradas en las necesidades de salud de los pobladores de São Cristóvão do Sul SC.

En un aspecto más general, el gran desafío enfrentado por el Grupo de Educación Permanente, fue la constitución de un equipo que se volviera efectivamente operativo, ya que la mayoría de los profesionales trabajaban en forma individual, centrados en su función específica, sin considerar el entorno de su universo de trabajo, principalmente, a los colegas involucrados. Trabajar los aspectos psico-afectivos implícitos y subjetivos en el proceso de trabajo, fue de gran valía en la creación de una identidad capaz de mantener y garantizar la unión del grupo. Problematizar ansiedades y miedos relacionados con el trabajo, fue fundamental para que los cambios pudieran acontecer de hecho.

A partir de la EPS, emergieron declaraciones sobre las dificultades de relacionarse con los superiores, situaciones particulares de cada integrante, diferencias, subjetividades y comprensiones diversas. 
La mantención de un espacio de discusión y modificación del proceso de gestión de la salud del municipio evidenciadas en las declaraciones de estos profesionales, guardan otras posibilidades de transformación, necesarias aún para el trabajo en el área de la salud de esta localidad.

Las irregularidades entre los principios del SUS y la forma como los servicios de salud del municipio están organizados, deja claro el vacío que separa la teoría de la práctica profesional. Las principales acciones se encuentran todavía concentradas, casi exclusivamente, en la Unidad Básica de Salud, en la atención individual a los enfermos y principalmente, en el médico. Este modelo de atención, hace que el exceso de demanda por consultas médicas, ocasione desgaste y provoque conflictos entre profesionales y los pobladores. Las discusiones realizadas demostraron que es posible re-pensar la atención en salud y la utilización de una estrategia diferente de aquella a la que están habituados.

Esta intervención buscó adoptar una perspectiva cualitativa de renovación y transformación de las prácticas profesionales, articulando los servicios a los saberes producidos en la academia. Para lo cual, orientó reflexiones acerca de la práctica profesional, fomentó discusiones, medió la solución de conflictos, contribuyó a la búsqueda de soluciones para los problemas cotidianos de la salud del referido municipio. Este trabajo posibilitó la construcción de un ambiente acogedor y democrático, facilitando la manifestación de angustias y dudas, garantizando el fortalecimiento del vínculo afectivo entre los participantes y permitiendo la mejoría del ambiente de trabajo.

La metodología de la EPS funcionó como una herramienta didáctica para la comprensión ampliada del proceso salud-enfermedad, contemplando además de los factores biológicos y psíquicos, la función de las condiciones sociales e individuales en la resolución de problemas. Los resultados de este trabajo evidencian la importancia de la integración enseñanza - servicio y la potencia de la EPS como estrategia para esta finalidad.

Se resalta que la extensión "Organização dos Serviços de Saúde: um Desafio da Educação Permanente" fue propuesta por el Grupo de Educación Permanente (EDUPE), bajo el sello del propio Curso y del Departamento de Ciencias Biológicas y de la Salud, incluido en 
el Proyecto de Asistencia Social de la UNIPLAC, y contó con el apoyo operacional y financiero del Municipio de São Cristóvão do Sul, teniendo su inicio en el año 2006, con término previsto para el año 2007, aun cuando los trabajos se extendieron hasta julio de 2008, en función de las necesidades y del compromiso de los gestores del municipio.

Se espera que esta intervención social haya contribuido no solamente a la capacitación profesional de un equipo de salud, sino que también, haya ampliado las posibilidades de reflexión acerca de la humanización de las prácticas en salud y de la organización del trabajo con base en metas discutidas colectivamente.

\section{Referencias Bibliográficas}

Brasil. Ministério da Saúde. Secretaria de Gestão do Trabalho e da Educação na Saúde. Departamento de Gestão da Educação na Saúde (2004). Política de educação $e$ desenvolvimento para o SUS: caminhos para a educação permanente em saúde - Pólos de Educação Permanente em Saúde. Brasília: EL MINISTERIO

Burg, R. (2005) Educação Permanente em Saúde: desafio ambicioso e necessário Interface - Comunic, Saúde, Educ, v.9.(16), pp.161-77.

Freire, P. (2000) Pedagogia da Autonomia: saberes necessários à prática educativa. São Paulo: Paz.

Morin, E. (2000) A cabeça bem-feita. Rio de Janeiro: Bertrand Brasil.

Komatsu, R. S. (1999) Aprendizagem Baseada em Problemas: um caminho para a transformação curricular. Revista Brasileira de Educação Médica, 23(2/3) PP.32-37.

Silveira, D. P.y Vieira, A. L. S. (2005) Reflexões sobre a ética do cuidado em saúde: desafios para a atenção psicossocial no Brasil. Estudos e pesquisas em psicologia, 5 (1), PP.

Silva Júnior, A.; Merhy, E.y Carvalho, L. (2003) Refletindo sobre o ato de cuidar da saúde. In: Pinheiro, R. e Mattos, R.A. Construção da integralidade: cotidiano, saberes $e$ práticas em saúde. Rio de Janeiro: ABRASCO. pp. 113-128.

Teixeira, C.F. (2003) A mudança do modelo de atenção à saúde no SUS: desatando nós, criando laços. Saúde em Debate, 27 (65), pp. 257-277. 
